\title{
Mathematical Model and Analysis of Corruption Dynamics with Optimal Control
}

\author{
Abayneh Kebede Fantaye ${ }^{1}$ and Zerihun Kinfe Birhanu $\mathbb{D}^{2}$ \\ ${ }^{1}$ Debre Tabor University, Department of Mathematics, Ethiopia \\ ${ }^{2}$ Hawassa University, Department of Mathematics, Ethiopia \\ Correspondence should be addressed to Zerihun Kinfe Birhanu; zerihunk@hu.edu.et
}

Received 22 September 2021; Revised 22 November 2021; Accepted 9 December 2021; Published 12 January 2022

Academic Editor: Bruno Carpentieri

\begin{abstract}
Copyright (c) 2022 Abayneh Kebede Fantaye and Zerihun Kinfe Birhanu. This is an open access article distributed under the Creative Commons Attribution License, which permits unrestricted use, distribution, and reproduction in any medium, provided the original work is properly cited.
\end{abstract}

\begin{abstract}
In this study, a deterministic mathematical model that explains the transmission dynamics of corruption is proposed and analyzed by considering social influence on honest individuals. Positivity and boundedness of solution of the model are proved and basic reproduction number $\left(R_{0}\right)$ is computed using the next-generation matrix method. The analysis shows that corruption-free equilibrium is locally and globally asymptotically stable whenever $R_{0}<1$. Also, the endemic equilibrium point is locally and globally asymptotically stable whenever $R_{0}>1$. Then, the model was extended to optimal control, and some numerical simulations with and without optimal control are also performed to verify the theoretical analysis using MATLAB. Numerical simulation of optimal control model shows that the prevention and punishment strategy is the most effective strategy to reduce the dynamic transmission of corruption.
\end{abstract}

\section{Introduction}

It is unconformable, profit gained by contradicting the law or rules, norms, or the action set in the community. The World Bank (WB) has defined corruption as the abuse of public office for private gain [1]. The Transparency International (TI) also defined corruption as the misuse of entrusted power for private benefit [2]. Corruption is colourless, shapeless, odourless, collusive, secret, stealthy, and shameless [3]. It is a worm within the body of the society and a cancer to economic, social, and political development [4]. Corruption involves bribes, illegal gratitude, conflict of interests, embezzlement of public funds, and economic extortion [5]. It is major cause of poverty around the world, especially in Africa [6] which suffers from deep-rooted corruption that hinders economic development, undermines democracy, and damages social justice and rule of law [7]. Yet, things are different in Ethiopia; its political systems are deficient in democratic power sharing, check and balance, accountable and transparent institution, and procedures [8]. It is one of the causes of instability and conflict which is seen in the present situation in Ethiopia [9].

The development of viable intervention strategies to mitigate corruption and corrupt practices requires a thorough understanding of the corrupt process and prevention and disengagement programs [10]. Mathematical modeling in epidemiology has become an effective tool for understanding and describing the dynamics of infectious disease [11]. The basic and important research subjects for epidemiological models are the existence of the threshold value which distinguishes whether the infectious disease will die out, the local and global stability of the disease-free and endemic equilibrium, the existence and extinction of the disease, etc. [12]. Several authors have studied the epidemiological corruption modeling approach as a disease. In particular, Gweryina et al. [13] derived and analyzed an epidemiological model of corruption with immunity clause in Nigeria. The simulation result shows that treating corruption in the presence of immunity clause regime will be a hard war to be won if the clause continued to exist. Shah et al. [14] formulate and 
analyze the nonlinear mathematical model, and in the upcoming year, the model may be helpful to society to reduce the burden of corruption, and punishment to the individual is a must for all irrespective of the position they held. Eguda et al. [7] formulated and analyzed a model with standard incidence that investigates the dynamics of corruption as a disease.

Hathroubi and Trabelsi [2] proposed a model on corruption dynamics. Based on the approximation of the honest population, an epidemiological corruption threshold was determined, but the stability analysis of both corruptionfree and endemic equilibria was not carried out. Crokidakis and Martins [15] proposed and analyzed a simple model of social contagion that represents the dynamics of social influences among politicians in an artificial corrupt parliament. The results show that there is a critical density of such zealot above which the honest deputies cannot survive as honest after a long time, while zealot density below the mentioned critical density, honest deputies coexist in the parliament with corrupt ones.

Nathan and Jackob [10] divided the total population into three compartments, susceptible $(S)$, corrupt $(C)$, and political corrupt $(M)$. Through media and campaigning from opposing parties such as anticorruption bodies, churches, and government political will to fight, the corrupt individuals $C(t)$ becomes susceptible $S(t)$. The model exhibits a threshold dynamics characterized by the basic reproduction $R_{0}$. When $R_{0}<1$, the system has a unique equilibrium point that is asymptotically stable. For $R_{0}>1$, the system has additional equilibrium point known as endemic which is globally asymptotically stable. They also assess strategies to counter corruption vice.

Abdulrahman [4] developed a deterministic mathematical model of corruption transmission dynamics as a disease. The basic reproduction number, corruption-free and endemic equilibrium point was determined. Numerical simulation was carried out and further revealed that corruption can only be removed to a manageable level but not totally eliminated. Legesse and Shiferaw [9] proposed and analyzed a mathematical model for the spread of corruption dynamics. The model is similar with characteristics to Abdulrahman [4] except that an individual who loses immunity gained through council in jail does not directly join corrupt class rather susceptible because of human behavior, and also, they are concerned with the impact of the awareness created by anticorruption.

Optimal control theory is a useful tool [16], for the corruption transmission dynamics and in decision-making processes regarding intervention programs for corruption control. Wang et al. [17] constructed optimal control model by the classic method of PMP (Pontryagin maximum principle) which is changing the general incidence rate into bilinear one and considering only discrete time delay for the sake of feasibility and simplicity on calculations. Athithan et al. [18] developed a deterministic model of the spread of corruption and its analysis using the differential equations. Then, the model extended to optimal control and the result shows that the level of corruption in the society can be reduced if corruption control efforts through media/punish- ments are increased and put in place. Danford et al. [6] developed a corruption dynamics model by modifying the work done by Athithan et al. [18] by including compartment of immune individuals with intervention strategies through combination of mass education and religious teaching.

All the above researchers have developed a mathematical model for the transmission of corruption dynamics as a disease. They considered a deterministic mathematical model by dividing the total population into susceptible, exposed, corrupt, infected, jailed, punished, honest, and recovered classes. For instance, Athithan et al. [18] considered susceptible, infected, and recovered classes and they extend into optimal control model, but the jailed and honest classes are not considered. Also, the model proposed by Legesse and Shiferaw [9] contains susceptible, corrupt, jailed, and honest classes. However, they did not consider the control strategies to minimize the spread of corruption. As a result, we motivate this study to fulfill the entire gap.

Our present model is different from the other, and it is that an honest individual can become susceptible, due to the effect of social influence. Furthermore, it extended to optimal control problem. This study is structured as follows. In Section 2, we develop our mathematical model for the transmission of corruption dynamics. We analyze the positivity and boundedness of solutions as well as stability of the corruption-free and endemic equilibria in Section 3. Section 4 deals about numerical simulation. Section 5 is the optimal control model. The formulation, existence, characterization, and numerical simulations of the optimal control model are also discussed. Finally, conclusion is given in Section 6.

\section{Model Formulation}

In this section, the total population is divided into four compartments: susceptible, corrupt, jailed, and honest individuals and we assumed that

(i) susceptible individuals are the innocent individuals who are not engaged in any corrupt activities or those jailed and then released after taking council

(ii) corrupt individuals are individuals who are engaged in corrupt activities and capable of influencing a susceptible individual to become corrupt

(iii) jailed corrupt individuals are individuals who are found to be guilty due to corrupt activities by law

(iv) honest individuals are truthful individuals that can never corrupt, but they become susceptible due to the pressure of social influence

(v) individuals can be corrupted only through contacts with corrupted individuals

(vi) susceptible, corrupt, and jailed individuals can be converted to honest individuals due to awareness created by anticorruption or counseling in jail, moral, media, and religious belief, and the impact 
Table 1: Parameters of the model.

\begin{tabular}{lc}
\hline Parameter & Description \\
\hline$\Lambda$ & Recruitment rate \\
$\delta$ & Effective corruption contact rate \\
$\theta$ & Rate at which corrupt individuals are caught and \\
$\gamma$ & imprisoned \\
$\mu$ & Maximum saturation of corrupt population \\
$\alpha$ & Proportion of individuals that joins $H$ from $S, C$, and $J$ \\
$\omega$ & Rate at which honest individuals become susceptible \\
\hline
\end{tabular}

of awareness is the same. The parameters of the model are presented in Table 1

Based on the flowchart of the model in Figure 1, we can describe the model as follows. There is a positive recruitment rate $\Lambda$ into the susceptible class and a positive natural death rate $\mu$ for all time under the study. Due to the influence of corrupt individuals, susceptible individuals can be corrupted at rate $(\beta C(t)) /(\theta+C(t))$. Furthermore, other model parameters are defined as follows: $\beta$ is the effective corruption contact rate, $\delta$ is the rate at which corrupt individuals are caught and imprisoned, $1 / \gamma$ is the average period jailed individuals spent in prison, $\alpha$ is the proportion at which susceptible $(S)$, corrupt $(C)$, and jailed $(J)$ individuals become honest $(H)$ due to awareness created by anticorruption or counseling in jail, media, moral, and religious belief, and $\omega$ is the rate at which the honest individuals $(H)$ become susceptible $(S)$ due to the pressure of social influence. Based on assumption and the flowchart for the corruption dynamics, we have the following system of nonlinear ordinary differential equations:

$$
\begin{aligned}
\frac{d S}{d t} & =\Lambda-\frac{\beta C S}{\theta+C}-(\mu+\alpha) S+\gamma(1-\alpha) J+\omega H, \\
\frac{d C}{d t} & =\frac{\beta C S}{\theta+C}-(\mu+\alpha+\delta) C, \\
\frac{d J}{d t} & =\delta C-(\mu+\gamma) J, \\
\frac{d H}{d t} & =\alpha(S+C+\gamma J)-(\mu+\omega) H,
\end{aligned}
$$

with

$$
\begin{aligned}
& S(0) \geq 0, \\
& C(0) \geq 0, \\
& J(0) \geq 0, \\
& H(0) \geq 0 .
\end{aligned}
$$

\section{Model Analysis}

In this section, we study the solution of (1) in the epidemiologically feasible region.

$$
\Omega=\left\{(S, C, J, H) \in R_{+}^{4}: 0 \leq S(t)+C(t)+J(t)+H(t) \leq \frac{\Lambda}{\mu}\right\} .
$$

3.1. Positivity and Boundedness of the Solution. Now, we study the positivity and boundedness of system of equation (1).

Theorem 1. Let the initial data be $\{S(0) \geq 0, C(0) \geq 0$, J(0) $\geq 0, H(0) \geq 0\} \in \Omega$, and then, the solution set $\{S(t), C(t), J$ $(t), H(t)\}$ of the system of equation (1) is nonnegative for all $t \geq 0$.

Proof. Now, let us start from the second equation of system (1) that

$$
\begin{aligned}
& \frac{d C}{d t}=\left(\frac{\beta S}{\theta+C}-(\mu+\alpha+\delta)\right) C, \\
& \frac{d C(t)}{d t}+\left((\mu+\alpha+\delta)-\frac{\beta S}{\theta+C}\right) C(t)=0, \\
& \frac{d C(t)}{d t}+F(t) C(t)=0,
\end{aligned}
$$

where $F(t)=(\mu+\alpha+\delta)-(\beta S /(\theta+C))$. Then, multiplying the above equation by integrating factor $e^{\int_{0}^{t} F(c) d c}$, we have

$$
\begin{aligned}
& \frac{d C(t)}{d t} e^{\int_{0}^{t} F(c) d c}+F(t) C(t) e^{\int_{0}^{t} F(c) d c}=0, \\
& \int_{0}^{t}\left[\frac{d}{d c}\left(C(c) e^{\int_{0}^{t} F(c) d c}\right)\right] d c=0, \\
& C(t)=C(0) e^{-\int_{0}^{t} F(c) d c} \geq 0 .
\end{aligned}
$$

From the third equation of system (1) that

$$
\begin{aligned}
& \frac{d J}{d t}=\delta C-(\mu+\gamma) J, \\
& \frac{d J(t)}{d t}+(\mu+\gamma) J(t) \geq 0,
\end{aligned}
$$

since from equation (5) we have $C(t) \geq 0$. Then, multiplying the above inequality by integrating factor $e^{\int_{0}^{t}(\mu+\gamma) d t}=e^{(\mu+\gamma) t}$, we have

$$
\begin{aligned}
\frac{d J(t)}{d t} e^{(\mu+\gamma) t}+(\mu+\gamma) J(t) e^{(\mu+\gamma) t} & \geq 0, \\
\int_{0}^{t}\left[\frac{d}{d t}\left(J(t) e^{(\mu+\gamma) t}\right)\right] d t & \geq 0, \\
J(t) \geq J(0) e^{-(\mu+\gamma) t} & \geq 0 .
\end{aligned}
$$




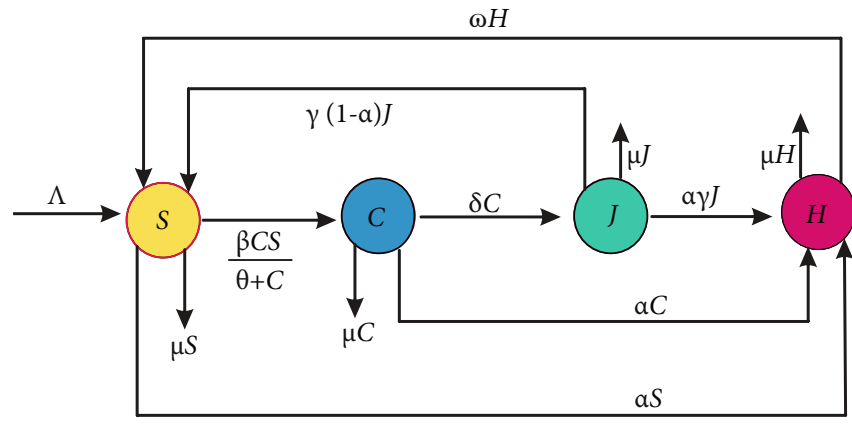

FIgURE 1: Flowchart for corruption dynamics.

Similarly, from the first and fourth equations of system (1), we can obtain that

$$
\begin{aligned}
S(t) & \geq S(0) e^{-\int_{0}^{t}\left(\frac{\beta C}{\theta+C}+\mu+\alpha\right) d s} \geq 0, \\
H(t) & \geq H(0) e^{-(\mu+\omega) t} \geq 0 .
\end{aligned}
$$

From the proofs of Theorem 1, we conclude that the model system of equation (1) is nonnegative.

Theorem 2. Assume that all the initial conditions are nonnegative in $R_{+}^{4}$ for the system

$$
\begin{aligned}
\Omega= & \left\{(S, C, J, H) \in R_{+}^{4} ; 0 \leq S(t)+C(t)\right. \\
& \left.+J(t)+H(t): N(t) \leq \frac{\Lambda}{\mu}\right\} .
\end{aligned}
$$

Then, the region $\Omega$ is positively invariant.

Proof. Differentiating the total population $N(t)=S(t)+C(t)$ $+J(t)+H(t)$ with respect to time and using system equation (1), we obtain

$$
\frac{d N}{d t}=\Lambda-\mu N
$$

By rearranging and then multiplying both sides of equation (10) by integrating factor $e^{\mu t}$ and after some simplification, we have

$$
N(t)=\frac{\Lambda}{\mu}+\left(N(0)-\frac{\Lambda}{\mu}\right) e^{-\mu t} .
$$

From (11), we have $\lim \sup _{t \rightarrow \infty} N(t) \leq \Lambda / \mu$. Thus, $\Omega$ is positively invariant (all solution in $\Omega$ remains in $\Omega$ for all time); that is, all solution of the model equation (1) with initial condition in $\Omega$ remains in $\Omega . \square$

Remark 3. In the region $\Omega$, our model of equation (1) is mathematically and epidemiologically well posed.

3.2. Corruption-Free Equilibrium Point of the Model. Corruption-free equilibrium (CFE) points $E_{0}$ are steady-state solutions, where there is no corruption in the society. In the absence of corruption, we have $C=0$ and $J=0$. Based on the of work Li et al. [19], the corruption-free equilibrium (CFE) points $E_{0}$ are given by

$$
E_{0}=(\widehat{S}, \widehat{C}, \widehat{J}, \widehat{H})=\left(\frac{\Lambda(\mu+\omega)}{(\mu+\alpha+\omega) \mu}, 0,0, \frac{\alpha \Lambda}{(\mu+\alpha+\omega) \mu}\right)
$$

The basic reproduction number $\left(R_{0}\right)$ : the basic reproduction number $R_{0}$ measures the expected number of secondary infections that result from one newly infected individual introduced into a susceptible population. Rewriting the system of equation (1) starting with the corrupt classes for both populations $C, J$ and then followed by uncorrupt classes $S, H$ from the two populations, the system of equation gives

$$
\begin{aligned}
\frac{d C}{d t} & =\frac{\beta C S}{\theta+C}-(\mu+\alpha+\delta) C, \\
\frac{d J}{d t} & =\delta C-(\mu+\gamma) J, \\
\frac{d S}{d t} & =\Lambda-\frac{\beta C S}{\theta+C}-(\mu+\alpha) S+\gamma(1-\alpha) J+\omega H, \\
\frac{d H}{d t} & =\alpha(S+C+\gamma J)-(\mu+\omega) H .
\end{aligned}
$$

Using the method of next-generation matrix, the Jacobian matrices at CFE for the transfer and new infection terms, respectively, are given by

$$
\begin{aligned}
& F=\left(\begin{array}{ll}
a & 0 \\
0 & 0
\end{array}\right), \\
& V=\left(\begin{array}{ll}
b & 0 \\
-\delta & d
\end{array}\right) .
\end{aligned}
$$

Then, the eigenvalues of $F V^{-1}$ is $\lambda_{1}=0, \lambda_{2}=a / b=(\beta \Lambda(\mu$ $+\omega)) /((\mu+\alpha+\omega)(\mu+\alpha+\delta) \mu \theta)$ where $a=(\beta \Lambda(\mu+\omega)) /$ $((\mu+\alpha+\omega) \theta \mu), b=\mu+\alpha+\delta$, and $d=\mu+\gamma$. The reproduction number $R_{0}$ is the dominant eigenvalue of $F V^{-1}$ or the spectral radius of $F V^{-1}$, i.e., $R_{0}=\rho\left(F V^{-1}\right)$, and hence, the 
reproduction number $R_{0}$ is given by

$$
R_{0}=\frac{\beta \Lambda(\mu+\omega)}{(\mu+\alpha+\omega)(\mu+\alpha+\delta) \mu \theta} .
$$

3.3. Corruption Endemic Equilibrium Point of the Model. Corruption will persist in the population, and the endemic equilibrium point of the model is given by $E_{1}=\left(S^{*}, C^{*}, J^{*}\right.$, $\left.H^{*}\right)$. The equations in the system (1) are solved as explained in Gumel [20] in terms of the effective corruption induced rate $(\beta C(t)) /(\theta+C(t))$ at equilibrium, given by

$$
\lambda^{*}=\frac{\beta C^{*}}{\theta+C^{*}} .
$$

Now, equation (1) can be rewritten as

$$
\begin{aligned}
\Lambda-\lambda^{*} S^{*}-e S^{*}+f J^{*}+\omega H^{*} & =0, \\
\lambda^{*} S^{*}-b C^{*} & =0, \\
\delta C^{*}-d J^{*} & =0, \\
\alpha\left(S^{*}+C^{*}+\gamma J^{*}\right)-g H^{*} & =0,
\end{aligned}
$$

where $b=\mu+\alpha+\delta, d=\mu+\gamma, e=\mu+\alpha, f=\gamma(1-\alpha), g=\mu$ $+\omega$.
From the second and third equations of system (17), we have

$$
\begin{aligned}
S^{*} & =\frac{b}{\lambda^{*}} C^{*}, \\
J^{*} & =\frac{\delta}{d} C^{*} .
\end{aligned}
$$

By substituting equation (18) in the first and last equations of (17), we obtain

$$
\begin{aligned}
& H^{*}=\frac{\left[b d \lambda^{*}+b d e-\lambda^{*} f \delta\right] C^{*}-\Lambda \lambda^{*} d}{\omega d \lambda^{*}}, \\
& H^{*}=\frac{\alpha b d+\lambda^{*}(\alpha d+\alpha \gamma \delta) C^{*}}{d g \lambda^{*}} .
\end{aligned}
$$

By comparing equations (19) and (20), we obtain

$$
C^{*}=\frac{\Lambda \lambda^{*} d g}{\lambda^{*}[b d g-\delta f g-\omega \alpha d-\omega \alpha \gamma \delta]+b d e g-\omega \alpha b d} .
$$

By substituting the values of $b, d, e, f$, and $g$ in equation (21), we obtain

$$
C^{*}=\frac{\Lambda \lambda^{*}(\mu+\omega)(\mu+\gamma)}{\lambda^{*}\left[\mu^{3}+(\omega+\gamma+\alpha+\delta) \mu^{2}+(\omega(\gamma+\delta)+\gamma \alpha(1+\delta)) \mu\right]+\left(\mu^{2}+(\omega+\alpha) \mu\right)(\mu+\alpha+\delta)(\mu+\gamma)} .
$$

Furthermore, substituting the values of $b, d, g$, and $C^{*}$ in equations (18) and (20), then we obtain

$$
\begin{aligned}
S^{*} & =\frac{\Lambda(\mu+\omega)(\mu+\gamma)(\mu+\alpha+\delta)}{\lambda^{*}\left[\mu^{3}+(\omega+\gamma+\alpha+\delta) \mu^{2}+(\omega(\gamma+\delta)+\gamma \alpha(1+\delta)) \mu\right]+\left(\mu^{2}+(\omega+\alpha) \mu\right)(\mu+\alpha+\delta)(\mu+\gamma)}, \\
J^{*} & =\frac{\delta \Lambda \lambda^{*}(\mu+\omega)}{\lambda^{*}\left[\mu^{3}+(\omega+\gamma+\alpha+\delta) \mu^{2}+(\omega(\gamma+\delta)+\gamma \alpha(1+\delta)) \mu\right]+\left(\mu^{2}+(\omega+\alpha) \mu\right)(\mu+\alpha+\delta)(\mu+\gamma)}, \\
H^{*} & =\frac{\alpha(\mu+\alpha+\delta)}{(\mu+\omega) \lambda^{*}}+\frac{\Lambda \lambda^{*} \alpha((\mu+\gamma)+\gamma \delta)}{\lambda^{*}\left[\mu^{3}+(\omega+\gamma+\alpha+\delta) \mu^{2}+(\omega(\gamma+\delta)+\gamma \alpha(1+\delta)) \mu\right]+\left(\mu^{2}+(\omega+\alpha) \mu\right)(\mu+\alpha+\delta)(\mu+\gamma)} .
\end{aligned}
$$

Next, substituting equation (22) into equation (16) and by simplifying, we have

$$
\begin{aligned}
\lambda^{*} & =\frac{(\mu+\alpha+\delta)(\mu+\alpha+\omega) \mu \theta(\beta \Lambda(\mu+\omega) /(\mu+\alpha+\omega)(\mu+\alpha+\delta) \mu \theta-1)(\mu+\gamma)}{\left(\mu^{2}+(\omega+\gamma+\alpha+\delta) \mu+\omega(\gamma+\delta)+\gamma \alpha(1+\delta)\right) \mu \theta+\Lambda(\mu+\omega)(\mu+\gamma)} \\
& =\frac{(\mu+\alpha+\delta)(\mu+\alpha+\omega) \mu \theta\left(R_{0}-1\right)(\mu+\gamma)}{\left(\mu^{2}+(\omega+\gamma+\alpha+\delta) \mu+\omega(\gamma+\delta)+\gamma \alpha(1+\delta)\right) \mu \theta+\Lambda(\mu+\omega)(\mu+\gamma)}
\end{aligned}
$$


Since, $R_{0}=(\beta \Lambda(\mu+\omega)) /((\mu+\alpha+\omega)(\mu+\alpha+\delta) \mu \theta)$ and note that all parameters are positive. Hence, if $R_{0}>1$, then $\lambda^{*}=\beta C^{*} /\left(\theta+C^{*}\right)>0$. This infers the existence of corruption in the specified population. With this, we can conclude the existence of corruption endemic equilibrium point.

3.4. Local Stability of the Corruption-Free Equilibrium Point. The local stability of the corruption-free equilibrium can be discussed by examining the linearized form of the system equation (1) at the steady state.

Theorem 4. The equilibrium solution $E_{0}$ of the nonlinear system of equation (1) is locally asymptotically stable, if $R_{0}<1$.

Proof. The Jacobian matrix of system equation (1) at corruption-free equilibrium point $E_{0}=((\Lambda(\mu+\omega)) /((\mu+\alpha$ $+\omega) \mu), 0,0, \alpha \Lambda /((\mu+\alpha+\omega) \mu))$ is

$$
J\left(E_{0}\right)=\left(\begin{array}{cccc}
-e & -a & f & \omega \\
0 & a-b & 0 & 0 \\
0 & \delta & -d & 0 \\
\alpha & \alpha & \alpha \gamma & -g
\end{array}\right)
$$

The characteristic equation of (25) at corruption-free equilibrium point $E_{0}$

$\left|J\left(E_{0}\right)-\lambda I_{4}\right|=\left|\begin{array}{cccc}-e-\lambda & -a & f & \omega \\ 0 & (a-b)-\lambda & 0 & 0 \\ 0 & \delta & -d-\lambda & 0 \\ \alpha & \alpha & \alpha \gamma & -g-\lambda\end{array}\right|=0$,

where $b=\mu+\alpha+\delta, d=\mu+\gamma, e=\mu+\alpha, f=\gamma(1-\alpha), g=\mu$ $+\omega, a=(\beta \Lambda(\mu+\omega)) /((\mu+\alpha+\omega) \theta \mu)$. Substituting the values of $a, b, d, e, f$, and $g$ in (26) and after some simplification, we obtain

$$
\begin{aligned}
& (-(\mu+\gamma)-\lambda)(\mu+\alpha+\delta) \\
& \cdot\left[\left(\frac{\beta \lambda(\mu+\omega)}{(\mu+\alpha+\delta)(\mu+\alpha+\omega) \mu \theta}-1\right)-\lambda\right] \\
& \cdot\left(\lambda^{2}+k_{1} \lambda+k_{2}\right)=0 .
\end{aligned}
$$

That is,

$$
(-(\mu+\gamma)-\lambda)(\mu+\alpha+\delta)\left[\left(R_{0}-1\right)-\lambda\right]\left(\lambda^{2}+k_{1} \lambda+k_{2}\right)=0
$$

Clearly,

$$
\begin{aligned}
& \lambda_{1}=-(\mu+\gamma)<0, \\
& \lambda_{2}=R_{0}-1 .
\end{aligned}
$$

Here, $\lambda_{2}<0$ if $R_{0}<1$, where $R_{0}=(\beta \lambda(\mu+\omega)) /((\mu+\alpha$ $+\delta)(\mu+\alpha+\omega) \mu \theta)$. Furthermore, using Routh Hurwitz criteria, the last equation of (28) has strictly negative real part since $k_{1}=(2 \mu+\alpha+\omega)>0$ and $k_{2}=\mu(\mu+\alpha+\omega)>0$. Therefore, our model of (1) at $E_{0}$ gives all eigenvalues possessing negative real part, and thus, it is locally asymptotical stable when $R_{0}<1$.

In the next section, we will find global stability of the corruption-free equilibrium point. Before we do this, we reduce the system of equation (1) by using equation (11), $H(t)=N(t)-S(t)-C(t)-J(t)=\Lambda / \mu+(N(0)-(\Lambda / \mu)) e^{-\mu t}$ $-S(t)-C(t)-J(t)$ to eliminate $H(t)$, from the first equation of system (1), which leads to the following reduced three-dimensional model:

$$
\begin{aligned}
\frac{d S}{d t}= & \frac{\Lambda}{\mu}(\mu+\omega)-\frac{\beta C S}{\theta+C}-(\mu+\alpha+\omega) S-\omega C \\
& +(\gamma(1-\alpha)-\omega) J+\omega\left(N(0)-\frac{\Lambda}{\mu}\right) e^{-\mu t}, \\
\frac{d C}{d t}= & \frac{\beta C S}{\theta+C}-(\mu+\alpha+\delta) C, \\
\frac{d J}{d t}= & \delta C-(\mu+\gamma) J,
\end{aligned}
$$

with initial condition $S(0) \geq 0, C(0) \geq 0, J(0) \geq 0$.

3.5. Global Stability of the Corruption-Free Equilibrium Point. To investigate the global stability, we apply the method proposed by Castillo-Chavez et al. [21]. According to Castillo-Chavez et al. [21], we rewrite the system of equation (30) in the following form:

$$
\begin{aligned}
& \frac{d X}{d t}=F(X, Y), \\
& \frac{d Y}{d t}=G(X, Y), G(X, 0)=0,
\end{aligned}
$$

where $X=(S)$ represent the number of uncorrupted classes, while $Y=(C, J)$ represent the number of corrupted classes with $X \in R$ and $Y \in R^{2}$, respectively. The corruption-free equilibrium point now is denoted by $E_{0}=\left(X^{0}, 0\right)$, where $X^{0}=\widehat{S}$. The conditions $\left(H_{1}\right)$ and $\left(H_{2}\right)$ are necessary conditions for the existence of global stability of the corruptionfree state: stable.

$\left(H_{1}\right)$ For $d X / d t=F(X, 0), X^{0}$ is globally asymptotically

$\left(H_{2}\right) G(X, Y)=A Y-\bar{G}(X, Y)$, where $\bar{G}(X, Y) \geq 0$ for $X, Y) \in \Omega$.

$A=D_{Y} G\left(X^{0}, 0\right)$ is an $M$ matrix (the off-diagonal elements of $A$ are nonnegative), and $\Omega$ is the region where the model makes biologically realistic. Then, CastilloChavez et al. [21] have shown that the following lemma is satisfied.

Lemma 5. If $R_{0}<1$, then the equilibrium point $E_{0}=\left(X^{0}, 0\right)$ of the system (30) is said to be globally asymptotically stable if the conditions $\left(H_{1}\right)$ and $\left(H_{2}\right)$ are satisfied. 
Now, we prove the following theorem.

Theorem 6. Suppose that $R_{0}<1$, then the corruption-free equilibrium point $E_{0}$ is globally asymptotically stable.

Proof. Let $X=(S)$ represent the number of uncorrupted classes, while $Y=(C, J)$ represent the number of corrupted classes, and $E_{0}=\left(X^{0}, 0\right)$, where $X^{0}=(\Lambda(\mu+\omega)) /((\mu+\alpha+\omega) \mu)$. Then, we have

$$
\begin{aligned}
\frac{d X}{d t}= & F(X, Y)=\frac{\Lambda}{\mu}(\mu+\omega)-\frac{\beta C S}{\theta+C}-(\mu+\alpha+\omega) S \\
& -\omega C+(\gamma(1-\alpha)-\omega) J+\omega\left(N(0)-\frac{\Lambda}{\mu}\right) e^{-\mu t}
\end{aligned}
$$

It is straightforward to see that at the corruption-free equilibrium point $S=\widehat{S}=(\Lambda(\mu+\omega)) /((\mu+\alpha+\omega) \mu), F(X, 0)$ $=0$. Thus,

$$
\begin{aligned}
& \frac{d X}{d t}=F(X, 0)=\frac{\Lambda}{\mu}(\mu+\omega)-(\mu+\alpha+\omega) X \\
& \quad+\omega\left(N(0)-\frac{\Lambda}{\mu}\right) e^{-\mu t}, \\
& \frac{d X}{d t}+(\mu+\alpha+\omega) X=\frac{\Lambda}{\mu}(\mu+\omega)+\omega\left(N(0)-\frac{\Lambda}{\mu}\right) e^{-\mu t}
\end{aligned}
$$

After some simplification, we can get that

$$
\begin{aligned}
X(t)= & \frac{\Lambda(\mu+\omega)}{(\mu+\alpha+\omega) \mu}+\left(X(0)-\frac{\Lambda(\mu+\omega)}{(\mu+\alpha+\omega) \mu}\right) e^{-(\mu+\alpha+\omega) t} \\
& +\omega\left(N(0)-\frac{\Lambda}{\mu}\right) e^{-\mu t}
\end{aligned}
$$

as $\quad t \longrightarrow \infty, X \longrightarrow X^{0}=\widehat{S}=(\Lambda(\mu+\omega)) /((\mu+\alpha+\omega) \mu)$. Hence, $X^{0}$ is globally asymptotically stable (that is, condition $H_{1}$ is satisfied)

Now, consider

$$
\begin{aligned}
A Y-\bar{G}(X, Y)= & \left(\begin{array}{cc}
\frac{\beta \widehat{S}}{\theta}-(\mu+\alpha+\delta) & 0 \\
\delta & -(\mu+\gamma)
\end{array}\right) \\
& \cdot\left(\begin{array}{c}
C \\
J
\end{array}\right)-\left(\begin{array}{cc}
\frac{\beta \widehat{S} C}{\theta}-\frac{\beta S C}{\theta+C} \\
0
\end{array}\right),
\end{aligned}
$$

where

$$
\begin{aligned}
A & =\left(\begin{array}{cc}
\frac{\beta \widehat{S}}{\theta}-(\mu+\alpha+\delta) & 0 \\
\delta & -(\mu+\gamma)
\end{array}\right), \\
\bar{G}(X, Y) & =\left(\begin{array}{c}
\frac{\beta \widehat{S} C}{\theta}-\frac{\beta S C}{\theta+C} \\
0
\end{array}\right) .
\end{aligned}
$$

From the system of equation (30), we consider $M(t)=$ $S(t)+C(t)+J(t)$ and differentiate it with respect to time; we have

$$
\frac{d M}{d t} \leq \frac{\Lambda}{\mu}(\mu+\omega)+\omega\left(N(0)-\frac{\Lambda}{\mu}\right) e^{-\mu t}-(\mu+\alpha+\omega) M .
$$

This implies that

$$
\frac{d M}{d t}+(\mu+\alpha+\omega) M \leq \frac{\Lambda}{\mu}(\mu+\omega)+\omega\left(N(0)-\frac{\Lambda}{\mu}\right) e^{-\mu t} .
$$

After rearranging equation (38), we have

$$
\begin{aligned}
M(t) \leq & \frac{\Lambda(\mu+\omega)}{\mu(\mu+\alpha+\omega)}-\frac{\Lambda(\mu+\omega)}{\mu(\mu+\alpha+\omega)} e^{-(\mu+\alpha+\omega) t} \\
& +\frac{\omega(\mu N(0)-\Lambda)}{\mu(\alpha+\omega)} e^{-\mu t}-\frac{\omega(\mu N(0)-\Lambda)}{\mu(\alpha+\omega)} e^{-(\mu+\alpha+\omega) t} \\
& +M(0) e^{-(\mu+\alpha+\omega) t}
\end{aligned}
$$

as $t \longrightarrow \infty, M(t) \leq(\Lambda(\mu+\omega)) /((\mu+\alpha+\omega) \mu)$. This implies that $M(t)$ is bounded by $\widehat{S}=(\Lambda(\mu+\omega)) /((\mu+\alpha+\omega) \mu)$, that is, $S \leq \widehat{S}$ and $\beta \widehat{S} C / \theta \geq \beta S C /(\theta+C)$. So $\bar{G}(X, Y) \geq 0$, and obviously, $A$ represent an $M$ matrix. Therefore, the conditions $\left(H_{1}\right)$ and $\left(H_{2}\right)$ are satisfied, so by Lemma 5 , the corruption-free equilibrium (CFE) $E_{0}$ is globally asymptotically stable provided that $R_{0}<1$.

3.6. Local Stability of Corruption Endemic Equilibrium Point. In this section, we apply the Jacobian stability approach to prove the local stability of corruption endemic equilibrium state.

Theorem 7. The corruption endemic equilibrium $E_{1}$ of the model is locally asymptotically stable when $R_{0}>1$.

Proof. Now, using the relation $H(t)=N(t)-(S(t)+C(t)+$ $J(t))$ allows as explained in Hethcote [22] and Benyah [23] to study the first three equations of the system (1). The Jacobian matrix of system (1) at $E_{1}$ is given by 


$$
J\left(E_{1}\right)=\left(\begin{array}{ccc}
-\frac{\left(\beta S^{*}+(\mu+\alpha+\omega)\left(\theta+C^{*}\right)\right.}{\left(\theta+C^{*}\right)} & -\frac{\beta \theta S^{*}+\omega\left(\theta+C^{*}\right)^{2}}{\left(\theta+C^{*}\right)^{2}} & \gamma(1-\alpha)-\omega \\
\frac{\beta C^{*}}{\theta+C^{*}} & -\frac{\left((\mu+\alpha+\delta)\left(\theta+C^{*}\right)^{2}-\beta \theta S^{*}\right)}{\left(\theta+C^{*}\right)^{2}} & 0 \\
0 & \delta & -(\mu+\gamma)
\end{array}\right) .
$$

From model equation of system (1) at corruption endemic equilibrium point, we have

$$
\begin{aligned}
(\mu+\alpha+\delta)\left(\theta+C^{*}\right) & =\beta S^{*}, \\
\mu+\gamma & =\frac{\delta C^{*}}{J^{*}}, \\
\mu+\omega & =\frac{\alpha\left(S^{*}+C^{*} \gamma J^{*}\right)}{H^{*}} .
\end{aligned}
$$

The characteristic equation at the endemic equilibrium point $E_{1}$ is given by

$$
\begin{aligned}
& (-A-\lambda)\left(-\frac{(A E+B D)}{A}-\lambda\right) \\
& \cdot\left(\left(-(\mu+\gamma)+\frac{\delta D(\gamma(1-\alpha)-\omega)}{A E+B D}\right)-\lambda\right)=0,
\end{aligned}
$$

where

$$
\begin{aligned}
& A=\frac{\left(\beta S^{*}+(\mu+\alpha+\omega)\left(\theta+C^{*}\right)\right.}{\left(\theta+C^{*}\right)}, \\
& B=\frac{\beta \theta S^{*}+\omega\left(\theta+C^{*}\right)^{2}}{\left(\theta+C^{*}\right)^{2}}, \\
& D=\frac{\beta C^{*}}{\theta+C^{*}}, \\
& E=\frac{\left((\mu+\alpha+\delta)\left(\theta+C^{*}\right)^{2}-\beta \theta S^{*}\right)}{\left(\theta+C^{*}\right)^{2}} .
\end{aligned}
$$

From equation (42), we obtain the following eigenvalues:

$$
\begin{aligned}
& \lambda_{1}=-A<0, \\
& \lambda_{2}=-\frac{(A E+B D)}{A}<0, \\
& \lambda_{3}=-(\mu+\gamma)+\frac{\delta D(\gamma(1-\alpha)-\omega)}{A E+B D} .
\end{aligned}
$$

For $\lambda_{3}$ to be negative, we must have

$$
\frac{A E+B D}{D}>\frac{\delta(\gamma(1-\alpha)-\omega)}{\mu+\gamma}
$$

Substituting the values of $A, B, D$, and $E$ from equations (43) and (44), we have

$$
\begin{gathered}
\frac{\left(\beta S^{*}+(\mu+\alpha+\omega)\left(\theta+C^{*}\right)\right)\left((\mu+\alpha+\delta)\left(\theta+C^{*}\right)^{2}-\beta \theta S^{*}\right)}{\beta C^{*}\left(\theta+C^{*}\right)} \\
+\frac{\beta \theta S^{*}+\omega\left(\theta+C^{*}\right)^{2}}{\left(\theta+C^{*}\right)^{2}}>\frac{\delta(\gamma(1-\alpha)-\omega)}{\mu+\gamma} .
\end{gathered}
$$

Using equation (41), $(\mu+\alpha+\delta)\left(\theta+C^{*}\right)^{2}-\beta \theta S^{*}=\beta C^{*}$ $S^{*}$, and using the value of $S^{*}=\left((\mu+\alpha+\delta) C^{*}\right) / \lambda^{*}$ and by multiplying both sides of equation (47) by $\mu+\gamma$, then equation (47) becomes

$$
\begin{aligned}
& \lambda^{*}\left[\frac{((\mu+\alpha+\delta)+(\mu+\alpha+\omega))\left(\left((\mu+\gamma)(\mu+\alpha+\delta) C^{*}\right) / \lambda^{*}\right)+\theta(\mu+\gamma)(\mu+\alpha+\delta)+\omega(\mu+\alpha+\delta)\left(\theta+C^{*}\right)}{\lambda^{*}\left(\theta+C^{*}\right)(\delta \gamma(1-\alpha))}\right]>1, \\
& \lambda^{*}>\frac{1}{\left(((\mu+\alpha+\delta)+(\mu+\alpha+\omega))\left(\left((\mu+\gamma)(\mu+\alpha+\delta) C^{*}\right) / \lambda^{*}\right)+\theta(\mu+\gamma)(\mu+\alpha+\delta)+\omega(\mu+\alpha+\delta)\left(\theta+C^{*}\right)\right) /\left(\lambda^{*}\left(\theta+C^{*}\right)(\delta \gamma(1-\alpha))\right)}>0 .
\end{aligned}
$$


TABLE 2: The parameter values of the model.

\begin{tabular}{lcc}
\hline Parameter & Value & Source \\
\hline$\Lambda$ & 12000 & Assumed \\
$\beta$ & 0.0234 & Legesse and Shiferaw [9] \\
$\delta$ & 0.000001 & Abdulrahman [4] \\
$\theta$ & 100000 & Legesse and Shiferaw [9] \\
$\gamma$ & 0.125 & Abdulrahman [4] \\
$\mu$ & 0.0160 & Legesse and Shiferaw [9] \\
$\omega$ & 0.0021 & Eguda et al. [7] \\
\hline
\end{tabular}

Therefore, $\lambda^{*}=\beta C^{*} /\left(\theta+C^{*}\right)>0$, and this holds whenever $R_{0}>1$ which is shown in (24). Hence, $\lambda_{3}<0$ if $R_{0}>1$ implying all the eigenvalues have negative real parts. Thus, $E_{1}$ is locally asymptotically stable.

3.7. Global Stability of Corruption Endemic Equilibrium Point. To establish the global asymptotical stability of corruption endemic equilibrium point $E_{1}$ of model of equation (1), we use the following results:

$$
\begin{aligned}
\Lambda=\frac{\beta C^{*} s^{*}}{\theta+C^{*}} & +(\mu+\alpha) S^{*}-\gamma(1-\alpha) J^{*} \omega H^{*}, \\
\frac{\beta C^{*} S^{*}}{\theta+C^{*}} & =(\mu+\alpha+\delta) C^{*}, \\
\delta C^{*} & =(\mu+\gamma) J^{*}, \\
\alpha\left(S^{*}+C^{*}+\gamma J^{*}\right) & =(\mu+\omega) H^{*} .
\end{aligned}
$$

Then, we can present and prove the following theorem.

Theorem 8. For $R_{0}>1$, the model equation of (1) at $E_{1}$ is global asymptotical stable for $\omega=0$.

Proof. We propose the following Lyapunov function for model of equation (1):

$$
\begin{aligned}
V(t)= & S-S^{*}-S^{*} \ln \frac{S}{S^{*}}+C-C^{*}-C^{*} \ln \frac{C}{C^{*}} \\
& +\frac{\beta C^{*} S^{*}}{\left(\theta+C^{*}\right) \delta C^{*}}\left(J-J^{*}-J^{*} \ln \frac{J}{J^{*}}\right) \\
& +\frac{\beta C^{*} S^{*}}{\alpha\left(\theta+C^{*}\right)\left(S^{*}+C^{*}+\gamma J^{*}\right)}\left(H-H^{*}-H^{*} \ln \frac{H}{H^{*}}\right) .
\end{aligned}
$$

By differentiating (50) with respect to time, we have

$$
\begin{aligned}
V^{\prime}(t)= & \left(1-\frac{S^{*}}{S}\right) \frac{d S}{d t}+\left(1-\frac{C^{*}}{C}\right) \frac{d C}{d t} \\
& +\frac{\beta C^{*} S^{*}}{\left(\theta+C^{*}\right) \delta C^{*}}\left(1-\frac{J^{*}}{J}\right) \frac{d J}{d t} \\
& +\frac{\beta C^{*} S^{*}}{\alpha\left(\theta+C^{*}\right)\left(S^{*}+C^{*}+\gamma J^{*}\right)}\left(1-\frac{H^{*}}{H}\right) \frac{d H}{d t} .
\end{aligned}
$$

Now,

$$
\begin{aligned}
& \left(1-\frac{S^{*}}{S}\right) \frac{d S}{d t}=\left(1-\frac{S^{*}}{S}\right)\left[\Lambda-\frac{\beta C S}{\theta+C}-(\mu+\alpha) S\right. \\
& +\gamma(1-\alpha) J+\omega H] \\
& =\left(1-\frac{S^{*}}{S}\right)\left[\frac{\beta C^{*} S^{*}}{\theta+C^{*}}+(\mu+\alpha) S^{*}\right. \\
& \left.-\gamma(1-\alpha) J^{*}-\omega H^{*}\right]-\left(1-\frac{S^{*}}{S}\right) \\
& \cdot\left[\frac{\beta C S}{\theta+C}-(\mu+\alpha) S+\gamma(1-\alpha) J+\omega H\right] \\
& \leq\left(1-\frac{S^{*}}{S}\right)\left[\frac{\beta C^{*} S^{*}}{\theta+C^{*}}\left(1-\frac{C S\left(\theta+C^{*}\right)}{C^{*} S^{*}(\theta+C)}\right)\right. \\
& \left.-\omega H\left(1-\frac{H}{H^{*}}\right)\right]=\frac{\beta C^{*} S^{*}}{\theta+C^{*}}\left[1-\frac{S^{*}}{S}\right. \\
& -\frac{C S\left(\theta+C^{*}\right)}{C^{*} S^{*}(\theta+C)}+\frac{C\left(\theta+C^{*}\right)}{C^{*}(\theta+C)} \\
& \left.-\omega H\left(1-\frac{H^{*}}{H}\right)\left(1-\frac{S^{*}}{S}\right)\right] \text {, } \\
& \left(1-\frac{C^{*}}{C}\right) \frac{d C}{d t}=\left(1-\frac{C^{*}}{C}\right)\left[\frac{\beta C S}{\theta+C}-(\mu+\alpha+\delta) C\right] \\
& =\left(1-\frac{S^{*}}{S}\right)\left[\frac{\beta C S}{\theta+C}-\frac{\beta C^{*} S^{*}}{\theta+C^{*}} \frac{C}{C^{*}}\right] \\
& =\frac{\beta C^{*} S^{*}}{\theta+C^{*}}\left[1+\frac{C S\left(\theta+C^{*}\right)}{C^{*} S^{*}(\theta+C)}-\frac{C}{C^{*}}-\frac{S\left(\theta+C^{*}\right)}{S^{*}(\theta+C)}\right],
\end{aligned}
$$

$$
\begin{gathered}
\frac{\beta C^{*} S^{*}}{\left(\theta+C^{*}\right) \delta C^{*}}\left(1-\frac{J^{*}}{J}\right) \frac{d J}{d t} \\
=\frac{\beta C^{*} S^{*}}{\left(\theta+C^{*}\right) \delta C^{*}}\left(1-\frac{J^{*}}{J}\right)[\delta C-(\mu+\gamma) J] \\
=\frac{\beta C^{*} S^{*}}{\left(\theta+C^{*}\right) \delta C^{*}}\left(1-\frac{J^{*}}{J}\right)\left[\delta C-\delta C^{*} \frac{J}{J^{*}}\right] \\
=\frac{\beta C^{*} S^{*}}{\left(\theta+C^{*}\right)}\left[1+\frac{C}{C^{*}}-\frac{J}{J^{*}}-\frac{J^{*} C}{J C^{*}}\right], \\
=\frac{\beta C^{*} S^{*}}{\alpha\left(\theta+C^{*}\right)\left(S^{*}+C^{*}+\gamma J^{*}\right)}\left(1-\frac{H^{*}}{H}\right) \frac{d H}{d t} \\
\cdot[\alpha(S+C+\gamma J)-(\mu+\omega) H] \\
=\frac{\left.\beta C^{*} S^{*}+C^{*}+\gamma J^{*}\right)}{\alpha\left(\theta+C^{*}\right)\left(S^{*}+C^{*}+\gamma J^{*}\right)}\left(1-\frac{H^{*}}{H}\right) \\
=\frac{\beta C^{*} S^{*}}{\left(\theta+C^{*}\right)}\left[1-\frac{H}{H^{*}}-\frac{(S+C+\gamma J)}{S^{*}+C^{*}+\gamma J^{*}}\left(\frac{H}{H}-1\right)\right] \\
{\left[\alpha(S+C+\gamma J)-\alpha\left(S^{*}+C^{*}+\gamma J^{*}\right) \frac{H}{H^{*}}\right]}
\end{gathered}
$$



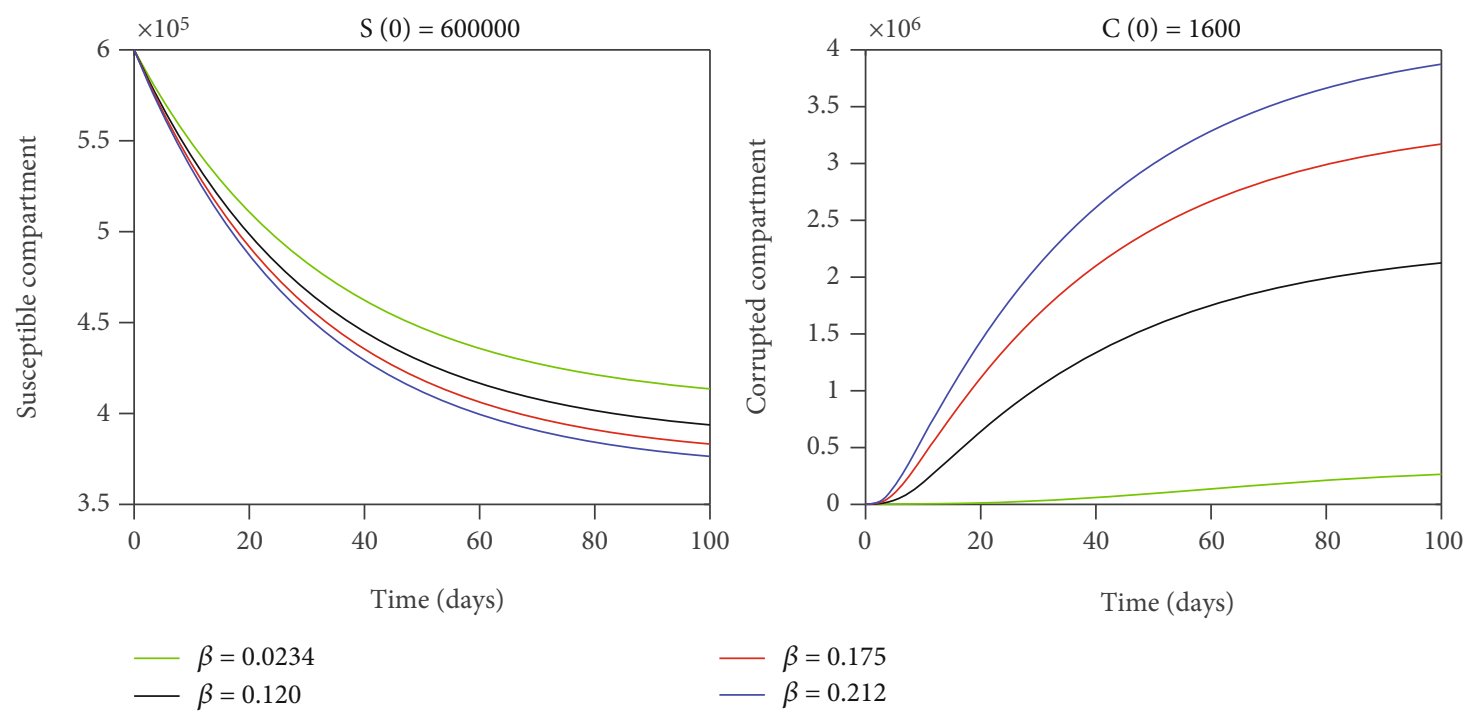

Figure 2: Susceptible and corrupted population with varying effective corruption contact rate, $\beta$.

By substituting the results from equations (52)-(55) into (51), we have

$$
\begin{aligned}
V^{\prime}(t)= & -\omega H^{*}\left(1-\frac{H^{*}}{H}\right)\left(1-\frac{S^{*}}{S}\right)+\frac{\beta C^{*} S^{*}}{\left(\theta+C^{*}\right)} \\
& \cdot\left[4-\frac{S^{*}}{S}-\frac{\left(\theta+C^{*}\right)}{(\theta+C)}\left(\frac{S}{S^{*}}-\frac{C}{C^{*}}\right)-\frac{J}{J^{*}}\right. \\
& \left.-\frac{J^{*} C}{J C^{*}}-\frac{H}{H^{*}}-\frac{(S+C+\gamma J)}{\left(S^{*}+C^{*}+\gamma J^{*}\right)}\left(\frac{H^{*}}{H}-1\right)\right] .
\end{aligned}
$$

Here, $V^{\prime}(t) \leq 0$ if

$$
\begin{gathered}
{\left[4-\frac{S^{*}}{S}-\frac{\left(\theta+C^{*}\right)}{(\theta+C)}\left(\frac{S}{S^{*}}-\frac{C}{C^{*}}\right)-\frac{J}{J^{*}}-\frac{J^{*} C}{J C^{*}}\right.} \\
\left.-\frac{H}{H^{*}}-\frac{(S+C+\gamma J)}{\left(S^{*}+C^{*}+\gamma J^{*}\right)}\left(\frac{H^{*}}{H}-1\right)\right] \leq 0 .
\end{gathered}
$$

Hence, using Lasalle [24], $E_{1}$ is global asymptotical stable whenever $R_{0}>1$. $\square$

Bifurcation: the center manifold theory is used to establish the stability behaviour of equilibrium point at $R_{0}=1$. The corruption-free equilibrium point changes its stability from stable to unstable at $R_{0}=1$ and there exists a positive equilibrium as $R_{0}$ crosses one. Furthermore, for $R_{0}=1, E_{0}$ $=E_{1}$. Thus, a transcritical bifurcation occurs in the model at the bifurcation point $R_{0}=1$.

\section{Numerical Simulation}

In this section, we verify our work numerically using MATLAB ODE solvers. Our simulations examine the effect of different combinations of parameters of the model on the transmission of the dynamics of corruption. The simulation is carried out by taking different values for the parameters. The set of parameter values whose sources are mainly from previous work as well as some assumption to investigate the effect of the honest individuals are susceptible to corruption in the dynamic transmission of corruption model. The simulations and analysis made are based on these initial conditions: $S(0)=600000, C(0)=1600, J(0)=0, H(0)=130000$ and the parameters values are displayed in Table 2.

From Figure 2, we observe that as effective corruption contact rate increases, the corrupted individuals increase, while the susceptible individuals decreased due to the influence of corrupted individuals. Furthermore, from Figure 3, we observe that as the proportion at which the susceptible, corrupt, and jailed individuals join the honest class increases, susceptible individuals decrease. However, as the proportion at which the susceptible, corrupt, and jailed individuals join the honest class decreases, corrupted individuals increased. From Figure 4, we observe that as the rate at which the corrupt individuals are caught and imprisoned due to their corrupt activities decreases, the corrupted individuals increased, while the jailed individuals decreased due to the secretive nature of corruption that means the corrupter individuals are remaining in the society without being arrested.

\section{Optimal Control Model}

In this study, we will minimize the objective functional, which will result in a balanced minimization of the goal and cost. The control that achieves this balance is called the optimal control, and the corresponding state is the optimal state.

5.1. Formulation of Optimal Control Model. In this section, we need to minimize the spread of corruption as well as minimize the cost associated with the control strategies. So, to achieve the goal of minimizing corruption dynamics, we reconsider the model (1) and formulate an optimal control model (58) to reduce the numbers of corruption. Hence, we use two control variables $u_{1}(t)$ and $u_{2}(t)$ to reduce the spread of corruption. The control $u_{1}(t)$ represents efforts intended to prevent corruption through education using 

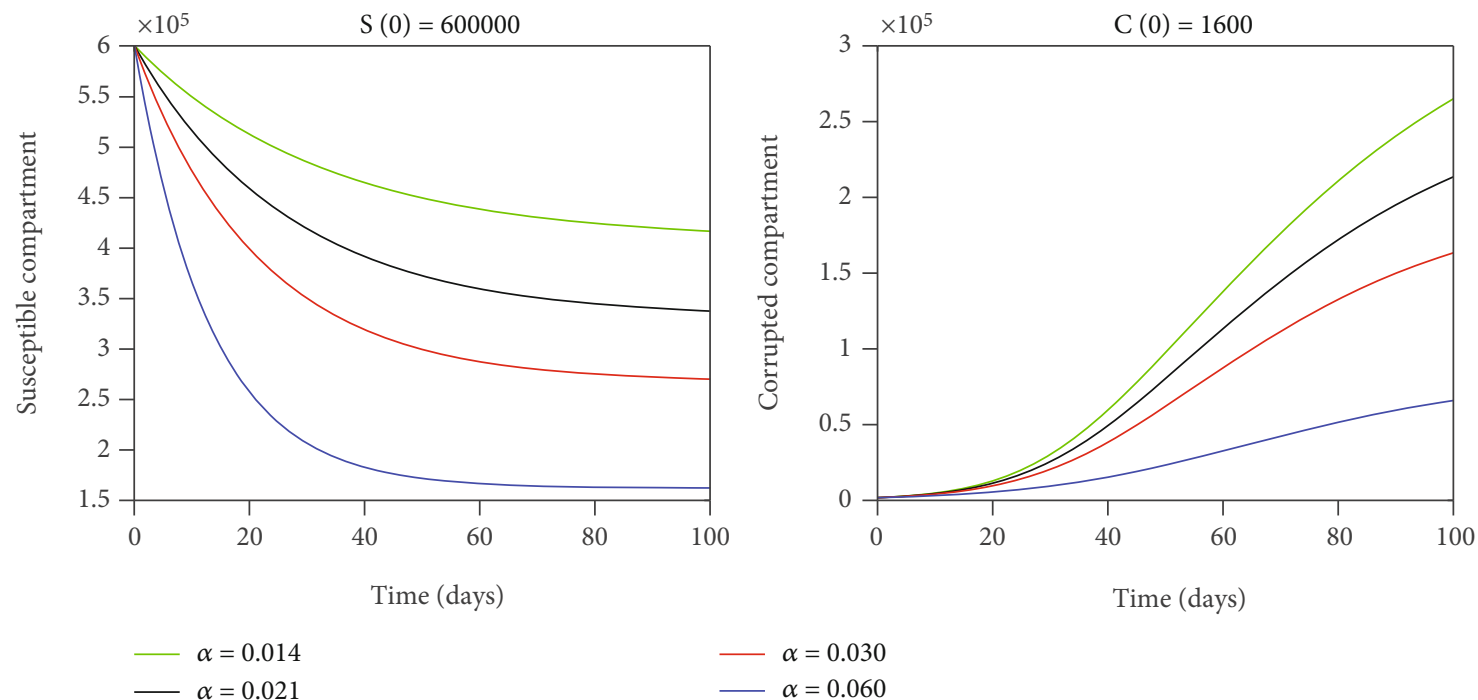

FigurE 3: Susceptible and corrupted population with varying proportion at which susceptible, corrupted, and jailed becomes honest, $\alpha$.
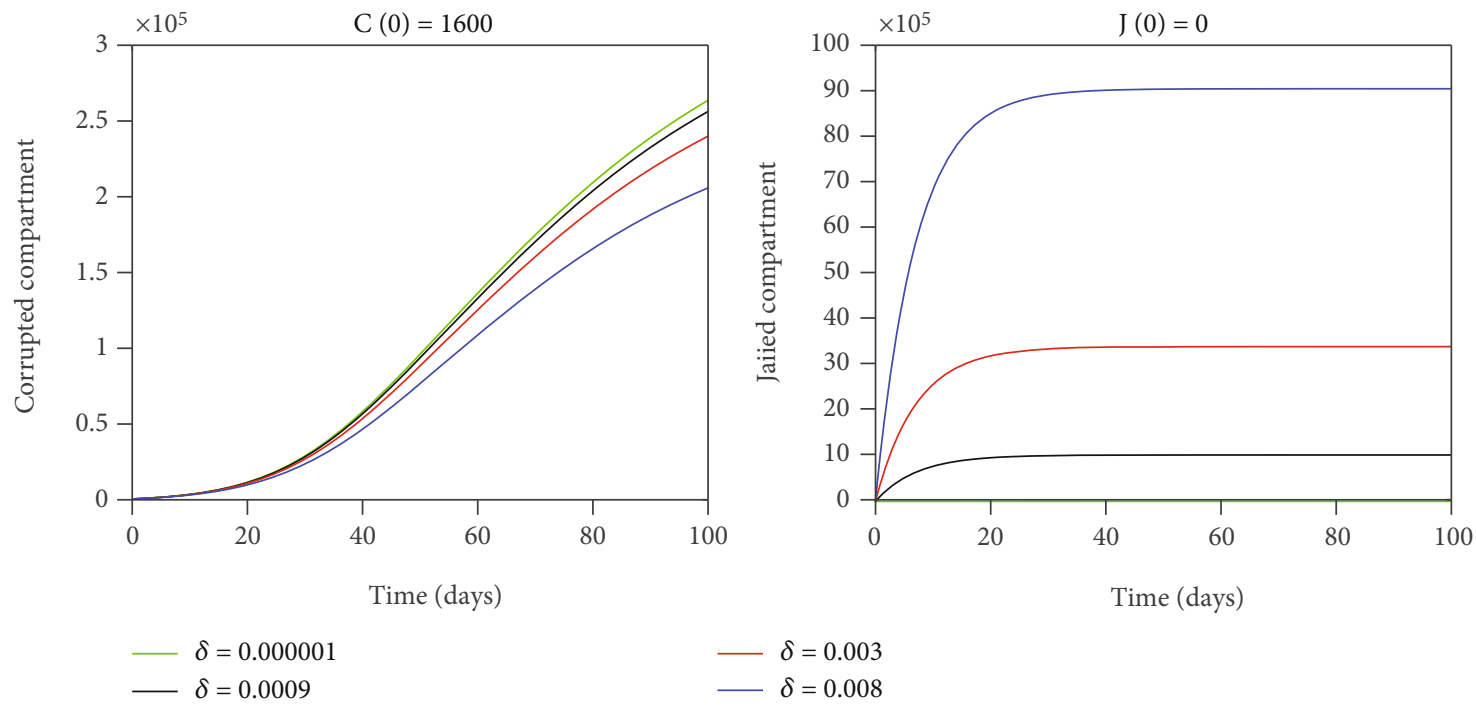

FIGURE 4: Corrupted and jailed population with varying rate at which corrupted individuals are caught and imprisoned, $\delta$.

social media, media, and civil society (religious organization); strong and efficient anticorruption policy is included. The control $u_{2}(t)$ represents the effort to encourage the punishment of corrupted individuals. The model with control is given by the following nonlinear system of ordinary differential equation:

$$
\begin{aligned}
& \frac{d S}{d t}=\Lambda-\left(1-u_{1}\right) \frac{\beta C S}{\theta+C}-(\mu+\alpha) S+\gamma(1-\alpha) J+\omega H, \\
& \frac{d C}{d t}=\left(1-u_{1}\right) \frac{\beta C S}{\theta+C}-(\mu+\alpha+\delta) C-u_{2} C, \\
& \frac{d J}{d t}=\delta C-(\mu+\gamma) J+u_{2} C, \\
& \frac{d H}{d t}=\alpha(S+C+\gamma J)-(\mu+\omega) H,
\end{aligned}
$$

with initial condition (2).
To study the optimal controls, the control set $U$, which is Lebesgue measurable, is defined as

$$
U=\left\{\left(u_{1}, u_{2}\right): 0 \leq u_{1} \leq 1,0 \leq u_{2} \leq 1,0 \leq t \leq t_{f}\right\} .
$$

The purpose of introducing controls in the model is to find the optimal level of the intervention strategy to reduce the spreads of the disease and the cost of implementation of the control. The control variables $u_{1}$ and $u_{2}$ are minimized subject to the differential equation (58), where we formulate the objective functional as

$$
J\left(u_{1}, u_{2}\right)=\int_{0}^{t_{f}}\left[A_{1} C+\frac{1}{2} A_{2} u_{1}^{2}+\frac{1}{2} A_{3} u_{2}^{2}\right] d t,
$$

where the constant $A_{i}, i=1,2,3$ are positive coefficients that balance the cost and the number of corruption at a time $t ; t_{f}$ 
is final time. After formulation of optimal control, the existence of control variables is proven. Then, use Pontryagin maximum principle that characterizes the control variable where an optimal solution of the model is obtained.

5.2. Existence of Optimal Control. In this section, we will study and analyze the existence of optimal control for the system of (58), and we can obtain the existence of optimal control using the result of Fleming and Rishel [25].

Theorem 9. There exists an optimal control $\left(u_{1}^{*}, u_{2}^{*}\right) \in U$ such that

$$
J\left(u_{1}^{*}, u_{2}^{*}\right)=\min _{\left(u_{1}, u_{2}\right) \in U} J\left(u_{1}, u_{2}\right)
$$

subject to control system (58) with initial condition (2).

Proof. Using a simplified version of an existence result [26] [27], the following conditions should be satisfied:

(i) The set of controls and the corresponding state variables are nonempty

(ii) The control set $U$ is convex and closed

(iii) The right-hand side of the state system is bounded by a linear function in the state and control variables

(iv) The integrand $L\left(t, S, C, J, H, u_{1}, u_{2}\right)$ of the objective functional is convex on $U$

(v) There exist constants $d_{1}, d_{2}>0$ and $k>1$ such that the objective functional is bounded below by $d_{1}$ $\left(\left|u_{1}\right|^{2}+\left|u_{2}\right|^{2}\right)^{k / 2}-d_{2}$

An existence result by Lukes [28] was used to give the existence of system of equation (58) with bounded coefficients, which gives condition (i). We note that our solutions are bounded. By definition, the control set is convex and closed. Since the state system is bilinear in $u_{1}, u_{2}$, the righthand side (58) satisfies condition (iii), using the boundedness of solution. The integrand of objective function (60) $A_{1} C(t)+(1 / 2) A_{2} u_{1}^{2}(t)+(1 / 2) A_{3} u_{2}^{2}(t)$ is clearly convex on $U$. Moreover, there exist constants $d_{1}, d_{2}>0$ and $k>1$ such that

$$
A_{1} C(t)+\frac{1}{2} A_{2} u_{1}^{2}(t)+\frac{1}{2} A_{3} u_{2}^{2}(t) \geq d_{1}\left(\left|u_{1}\right|^{2}+\left|u_{2}\right|^{2}\right)^{k / 2}-d_{2}
$$

since the state variable is bounded. We can conclude that there exists an optimal control pair. This ends the proof. $\square$

5.3. Characterization of Optimal Control. In this section, we will use Pontryagin maximum principle (PMP) to obtain the optimal control that satisfies the necessary conditions. This principle used to convert (58) and (60) into a problem of minimizing pointwise Hamiltonian $H$ problem with respect to control $u_{1}(t)$ and $u_{2}(t)$.

Theorem 10. Consider an optimal control $u^{*}=\left(u_{1}^{*}, u_{2}^{*}\right) \in U$ and solution of $S^{*}(t), C^{*}(t), J^{*}(t), H^{*}(t)$ with the corresponding state system (58) and (60) that minimizes $J\left(u_{1}, u_{2}\right)$ over $U$. Then, there exist adjoint variables $\lambda_{1}(t), \lambda_{2}(t), \lambda_{3}(t)$, and $\lambda_{4}(t)$ satisfying

$$
\begin{aligned}
\frac{d \lambda_{1}}{d t}= & \frac{\beta C^{*}}{\theta+C^{*}}\left(1-u_{1}\right)\left(\lambda_{1}-\lambda_{2}\right)+(\mu+\alpha) \lambda_{1}-\alpha \lambda_{4}, \\
\frac{d \lambda_{2}}{d t}= & \frac{\beta S^{*} \theta}{\left(\theta+C^{*}\right)^{2}}\left(1-u_{1}\right)\left(\lambda_{1}-\lambda_{2}\right) \\
& +\left(\mu+\alpha+\delta+u_{2}\right) \lambda_{2}-\left(\delta+u_{2}\right) \lambda_{3} \\
& -\alpha \lambda_{4}-A_{1}, \\
\frac{d \lambda_{3}}{d t}= & (\mu+\gamma) \lambda_{3}-\gamma(1-\alpha) \lambda_{1}-\alpha \gamma \lambda_{4}, \\
\frac{d \lambda_{4}}{d t}= & (\mu+\omega) \lambda_{4}-\omega \lambda_{1},
\end{aligned}
$$

with transversality conditions:

$$
\lambda_{1}\left(t_{f}\right)=\lambda_{2}\left(t_{f}\right)=\lambda_{3}\left(t_{f}\right)=\lambda_{4}\left(t_{f}\right)=0
$$

Moreover, for $t \in\left[0, t_{f}\right]$, the optimal controls $u_{1}^{*}$ and $u_{2}^{*}$ are given by

$$
\begin{aligned}
& u_{1}^{*}=\min \left\{1, \max \left(0, \frac{\left(\lambda_{2}-\lambda_{1}\right) \beta C^{*} S^{*}}{A_{2}\left(\theta+C^{*}\right)}\right)\right\}, \\
& u_{2}^{*}=\min \left\{1, \max \left(0, \frac{\left(\lambda_{2}-\lambda_{3}\right) C^{*}}{A_{3}}\right)\right\} .
\end{aligned}
$$

Proof. The Hamiltonian equation is given by

$$
\begin{aligned}
H\left(t, x, u^{*}, \lambda\right)= & A_{1} C^{*}(t)+\frac{1}{2} A_{2} u_{1}^{2^{*}}(t)+\frac{1}{2} A_{3} u_{2}^{2^{*}}(t) \\
& +\lambda_{1}\left[\Lambda-\left(1-u_{1}^{*}\right) \frac{\beta C^{*} S^{*}}{\theta+C^{*}}-(\mu+\alpha) S^{*}\right. \\
& \left.+\gamma(1-\alpha) J^{*}+\omega H^{*}\right]+\lambda_{2}\left[\left(1-u_{1}^{*}\right) \frac{\beta C^{*} S^{*}}{\theta+C^{*}}\right. \\
& \left.-(\mu+\alpha+\delta) C^{*}-u_{2} C^{*}\right]+\lambda_{3}\left[\delta C^{*}\right. \\
& \left.-(\mu+\gamma) J^{*}+u_{2} C^{*}\right]+\lambda_{4}\left[\alpha\left(S^{*}+C^{*}+\gamma J^{*}\right)\right. \\
& \left.-(\mu+\omega) H^{*}\right] .
\end{aligned}
$$

The adjoint equation and the transversality conditions can be obtained by Pontryagin's maximum principle which is given in Boltyanskiy et al. [29]. Now, we use Hamiltonian (66) and differentiating (66) with respect to $S, C, J$, and $H$, respectively, we get 


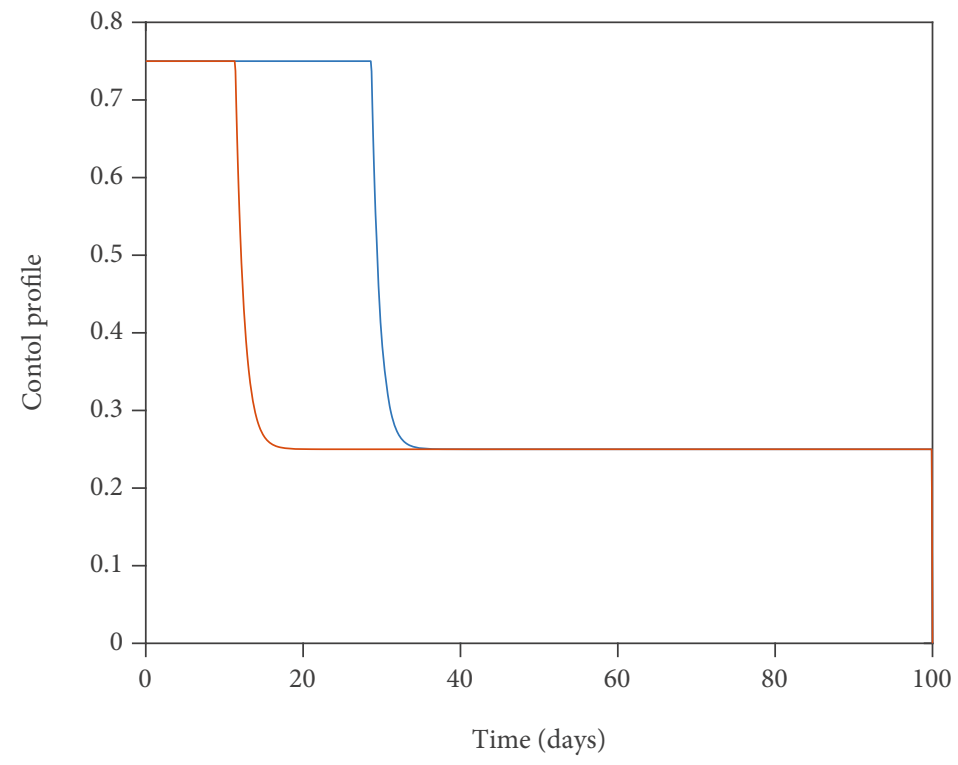

Prevention, $\mathrm{u}_{1}$

Punishment, $\mathrm{u}_{2}$

FigURE 5: Simulation showing the profile for the optimal control $u_{1}$ and $u_{2}$.
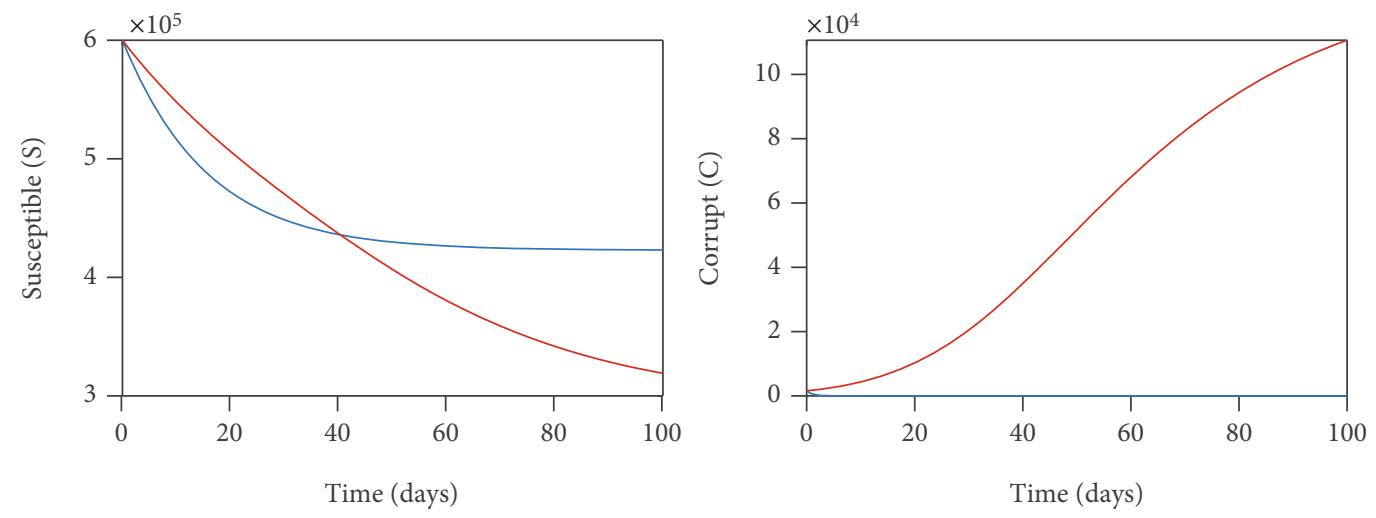

- S with optimal control

_ $S$ with out optimal control

- C with optimal control

C with out optimal control
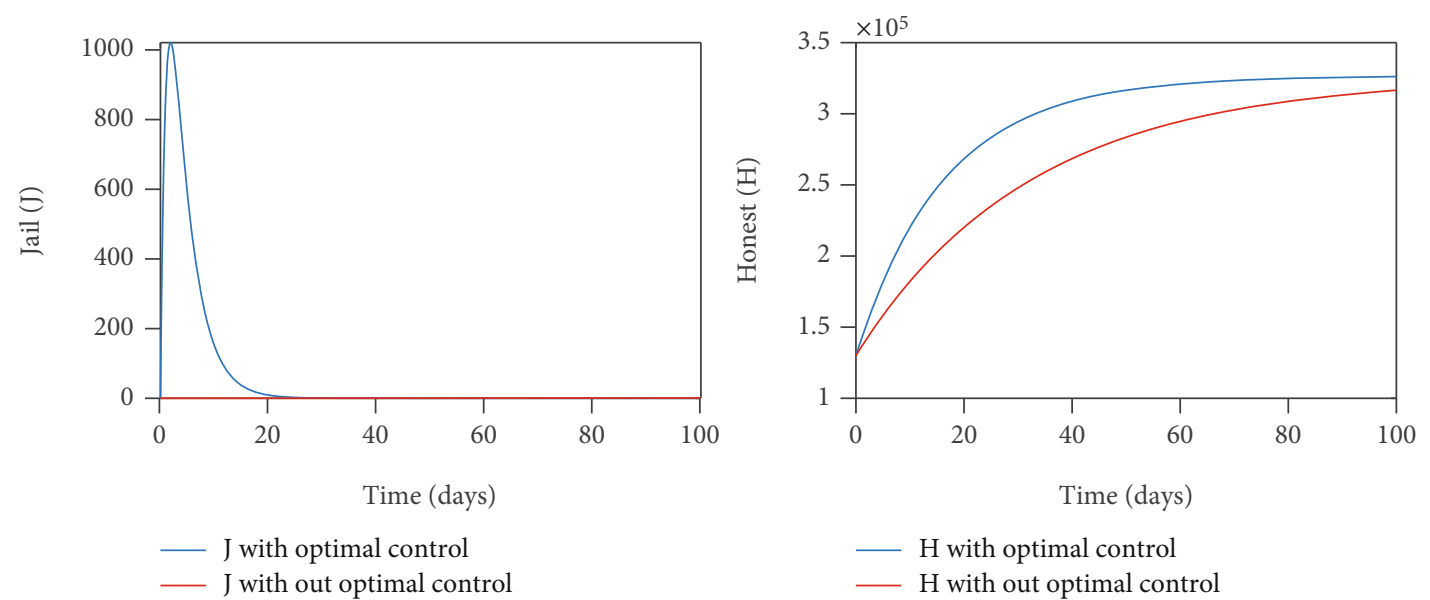

Figure 6: Simulation showing the optimal solution for susceptible, corrupt, jailed, and honest via use of $u_{1}$ and $u_{2}$ when $\beta=0.0234, \delta=$ 0.000001 , and $\alpha=0.014$. 

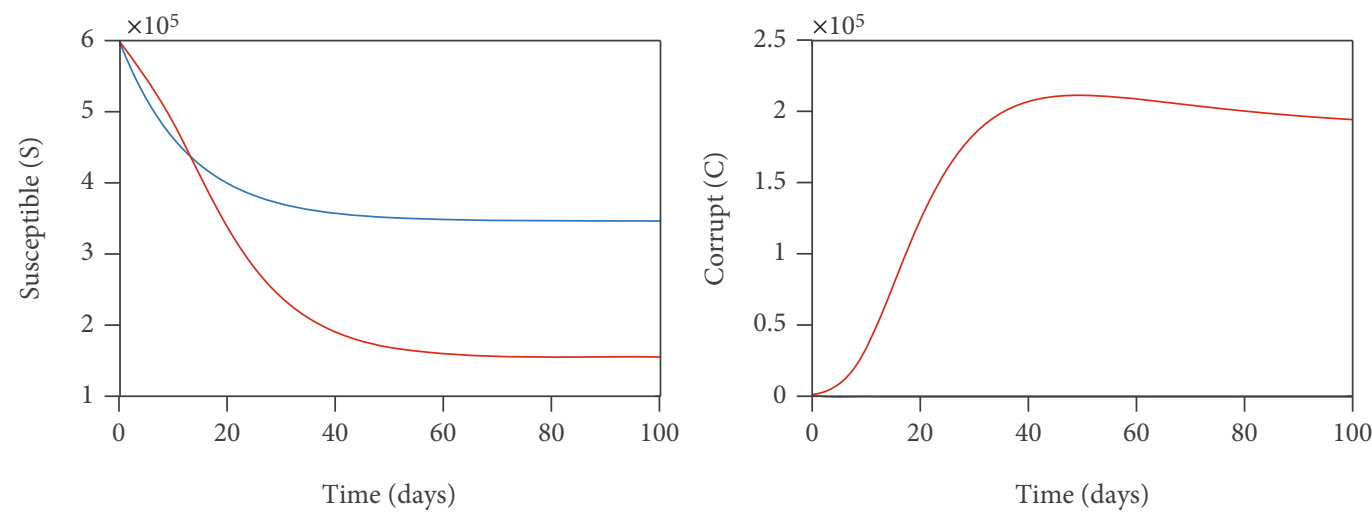

- S with optimal control

— S with out optimal control

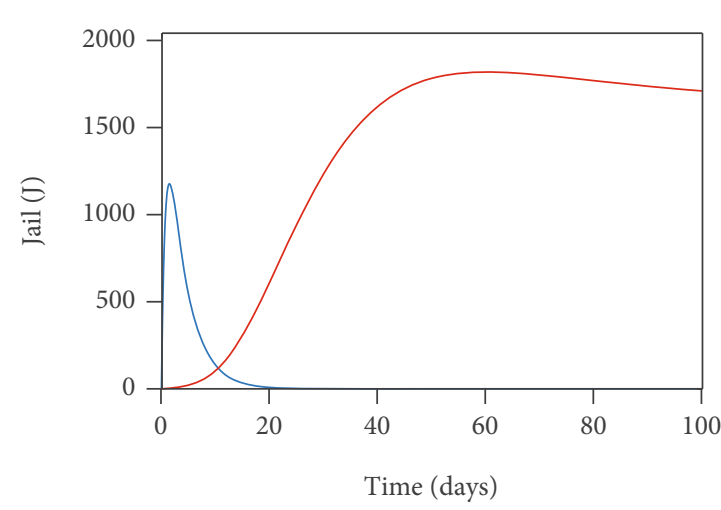

- J with optimal control

_ J with out optimal control

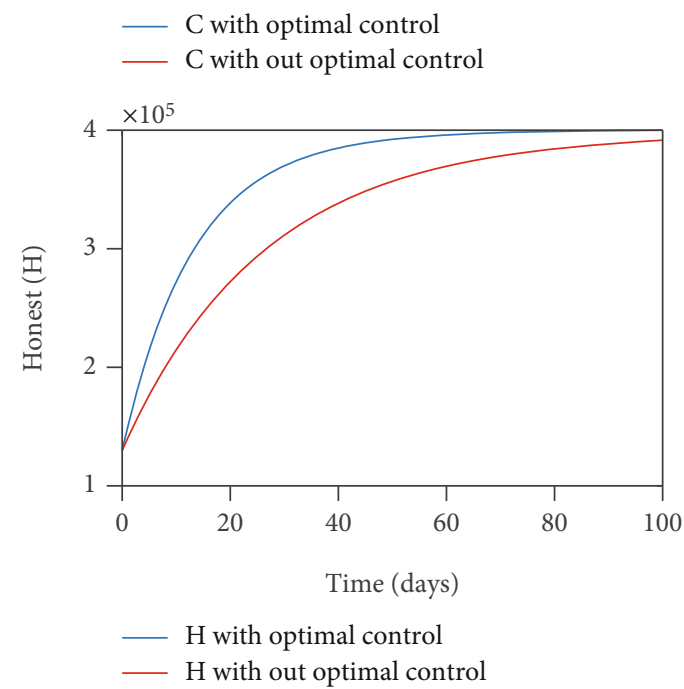

FIGURE 7: Simulation showing the optimal solution for susceptible, corrupt, jailed, and honest via use of $u_{1}$ and $u_{2}$ when $\beta=0.07, \delta=0.0012$, and $\alpha=0.021$

$$
\begin{aligned}
\frac{d \lambda_{1}}{d t}= & \frac{-\partial H}{\partial S^{*}(t)}=\frac{\beta C^{*}}{\theta+C^{*}}\left(1-u_{1}^{*}\right)\left(\lambda_{1}-\lambda_{2}\right)+(\mu+\alpha) \lambda_{1}-\alpha \lambda_{4}, \\
\frac{d \lambda_{2}}{d t}= & \frac{-\partial H}{\partial C^{*}(t)}=\frac{\beta S^{*} \theta}{\left(\theta+C^{*}\right)^{2}}\left(1-u_{1}^{*}\right)\left(\lambda_{1}-\lambda_{2}\right) \\
& +\left(\mu+\alpha+\delta+u_{2}^{*}\right) \lambda_{2}-\left(\delta+u_{2}\right) \lambda_{3}-\alpha \lambda_{4}-A_{1}, \\
\frac{d \lambda_{3}}{d t}= & \frac{-\partial H}{\partial J^{*}(t)}=(\mu+\gamma) \lambda_{3}-\gamma(1-\alpha) \lambda_{1}-\alpha \gamma \lambda_{4}, \\
\frac{d \lambda_{4}}{d t}= & \frac{-\partial H}{\partial H^{*}(t)}=(\mu+\omega) \lambda_{4}-\omega \lambda_{1},
\end{aligned}
$$

with $t_{1}\left(t_{f}\right)=0, t_{2}\left(t_{f}\right)=0, t_{3}\left(t_{f}\right)=0$, and $t_{4}\left(t_{f}\right)=0$. Next, by using optimality condition, we can find the optimal controls $u_{1}^{*}(t)$ and $u_{2}^{*}(t)$ for $t \in\left[0, t_{f}\right]$.

$$
\begin{gathered}
\frac{\partial H}{\partial u_{1}}=0, \text { at } u_{1}=u_{1}^{*}, \\
\frac{\partial H}{\partial u_{2}}=0, \text { at } u_{2}=u_{2}^{*} .
\end{gathered}
$$

Now,

$$
\begin{aligned}
\frac{\partial H}{\partial u_{1}^{*}} & =\frac{\partial H}{\partial u_{1}^{*}}=A_{2} u_{1}^{*}+\frac{\beta C S}{\theta+C} \lambda_{1}-\frac{\beta C S}{\theta+C} \lambda_{2}=0, \\
u_{1}^{*} & =\frac{\beta C S\left(\lambda_{2}-\lambda_{1}\right)}{(\theta+C) A_{2}}, \\
\frac{\partial H}{\partial u_{2}^{*}} & =\frac{\partial H}{\partial u_{2}^{*}}=A_{3} u_{2}^{*}-C \lambda_{2}+C \lambda_{3}=0, \\
u_{2}^{*} & =\frac{C^{*}\left(\lambda_{2}-\lambda_{3}\right)}{A_{3}} .
\end{aligned}
$$

By standard control arguments involving the bounds in $U$ controls, we can simply obtain $u_{1}^{*}$ and $u_{2}^{*}$ in the form of equation (65). That is

$$
\begin{aligned}
& u_{1}^{*}=\min \left\{1, \max \left(0, \frac{\left(\lambda_{2}-\lambda_{1}\right) \beta C^{*} S^{*}}{A_{2}\left(\theta+C^{*}\right)}\right)\right\}, \\
& u_{2}^{*}=\min \left\{1, \max \left(0, \frac{\left(\lambda_{2}-\lambda_{3}\right) C^{*}}{A_{3}}\right)\right\} .
\end{aligned}
$$

Hence, the optimal control pair is characterized. $\square$ 
5.4. Numerical Simulation of Optimal Control Model. In this section, we conduct numerical simulation in order to investigate the effects of the control strategies such as prevention and punishment to reduce the spread of corruption. The optimality system is a nonlinear two-point boundary value problem, with separated boundary conditions at times $t=0$ and $t=t_{f}$.

The constant cost coefficients corresponding to control measures are also estimated in the objective function as stated: $A_{1}=1000, A_{2}=0.000001, A_{3}=100$. The parameter values used in the simulation of this model are also stated in Table 2 with the set initial conditions: $S(0)=100, C(0)$ $=1600, J(0)=0$, and $H(0)=130000$, and we consider the entire period $t_{f}=100$ days.

5.4.1. Optimal Control Using Prevention and Punishment. In these scenarios, the use of prevention strategies $u_{1}$ and punishment strategies $u_{2}$ was applied to optimize the cost functional $J$. The profile of optimal controls $u_{1}$ and $u_{2}$ is shown in Figure 5.

From Figure 5, we observe that to control the spread of corruption in 100 days, the use of prevention and punishment strategies must be hold effectively. By applying the optimal control $u_{1}$ and $u_{2}$ and when some parameter value are varying, the dynamics of susceptible, corrupt, jailed, and honest are shown in Figures 6 and 7. We detect that due to the control strategies and due to increasing effect of corruption contact rate $(\beta)$, the rate at which corrupt individuals are caught and imprisoned $(\delta)$, and the proportion at which susceptible, corrupt, and jailed join the honest class $(\alpha)$ applied, susceptible individuals are slightly decreasing with optimal control compared to susceptible without optimal control. From Figures 6 and 7, corrupt individuals increase at a fast rate without control strategies but decrease at a fast rate with optimal control strategies. Furthermore, we observe that jailed individuals increase with optimal control, but it will decrease after 20 days, while it increases slightly without optimal control. Honest individuals increase at a fast rate with optimal control than honest without optimal control strategies.

\section{Conclusion}

In this study, a mathematical model on the transmission dynamics of corruption was formulated. We first showed that there exists a domain where the model is epidemiological and mathematically well posed. The reproductive number, $R_{0}$, corruption-free and endemic equilibrium points are computed. Based on the value of the basic reproduction number, $R_{0}$, the stability of the corruption-free and endemic equilibrium points is investigated. If $R_{0}<1$, then the corruption-free equilibrium point $E_{0}$ is locally and globally asymptotically stable (i.e., corruption decreases from the community over a period of time). We also showed that an endemic equilibrium point $E_{1}$ exists and is locally asymptotically stable for all $R_{0}>1$ (i.e., corruption persists in the community). The analysis and the numerical simulations without control show that as the effective corruption contact rate decreases, corrupt individuals decrease. Furthermore, as the proportion at which susceptible, corrupt, and jailed individuals become honest as well as the rate at which the corrupt individuals are caught and imprisoned increases, corrupt individuals decrease. Otherwise, corruption spreads rapidly in the society. Then, the model is extended to include two time-dependent control variables; prevention $u_{1}$ and punishment $u_{2}$. The study established and proved the existence and characterization of an optimal control solution. Numerical simulations of optimal control (OC) were carried out using MATLAB. The result shows that the two strategies, the prevention $u_{1}$ and punishment $u_{2}$ control strategies, are the most effective to reduce the spread of corruption in the population. The cost-effectiveness analysis of the integrated strategy would be considered in a future study.

\section{Data Availability}

The data used to support the findings of this study are included in the article. Actually, we used data from other papers for the simulation. The papers are properly cited.

\section{Conflicts of Interest}

The authors declare that they have no conflicts of interest.

\section{References}

[1] World Bank, Helping Countries Combat Corruption: The Role of the World Bank, World Bank, 1997.

[2] S. Hathroubi and H. Trabelsi, "Epidemic corruption: a bioeconomic homology," European Scientific Journal, vol. 10, pp. 228-235, 2014.

[3] G. Caiden, "Dealing with administrative corruption," in Handbook of administrative ethics, CRC Press, 2001.

[4] S. Abdulrahman, "Stability analysis of the transmission dynamics and control of corruption," Pacific Journal of Science and Technology, vol. 15, no. 1, pp. 99-113, 2014.

[5] A. Binuyo, "Eigenvalue elasticity and sensitivity analyses of the transmission dynamic model of corruption," Journal of Nigerian Society of Physical Sciences, vol. 1, pp. 30-34, 2019.

[6] O. Danford, M. Kimathi, and S. Mirau, "Mathematical modelling and analysis of corruption dynamics with control measures in Tanzania," Mathematics and Informatics, vol. 19, pp. 57-79, 2020.

[7] F. Eguda, F. Oguntolu, and T. Ashezua, "Understanding the dynamics of corruption using mathematical modelling approach," International Journal of Innovative Science, Engineering \& Technology, vol. 4, no. 8, pp. 190-197, 2017.

[8] G. Kebede, "Political corruption: political and economic state capture in Ethiopia," European Scientific Journal, vol. 9, no. $35,2013$.

[9] L. Legesse and F. Shiferaw, "Modelling corruption dynamics and its analysis," Ethiopian Journal of Sciences and Sustainable Development, vol. 5, no. 2, pp. 13-27, 2018.

[10] O. Nathan and K. Jackob, "Stability analysis in a mathematical model of corruption in Kenya," Asian Research Journal of Mathematics, vol. 15, no. 4, pp. 1-15, 2019.

[11] K. Hattaf, "A new generalized definition of fractional derivative with non-singular kernel," Computation, vol. 8, no. 2, p. $49,2020$. 
[12] K. Hattaf, A. A. Lashari, Y. Louartassi, and N. Yousfi, "A delayed sir epidemic model with a general incidence rate," Electronic Journal of Qualitative Theory of Differential Equations, vol. 2013, pp. 1-9, 2013.

[13] R. I. Gweryina, M. Y. Kura, and E. Okwu, "An epidemiological model of corruption with immunity clause in Nigeria," World Journal of Modelling and Simulation, vol. 15, no. 3, pp. 262275, 2019.

[14] N. H. Shah, B. M. Yeolekar, and Z. A. Patel, "Epidemics of corruption using incidence function," Economic Computation \& Economic Cybernetics Studies \& Research, vol. 51, no. 2, 2017.

[15] N. Crokidakis and J. S. Sá Martins, "Can honesty survive in a corrupt parliament?," International Journal of Modern Physics C, vol. 29, no. 10, p. 1850094, 2018.

[16] J.-Q. Zhao, E. Bonyah, B. Yan et al., "A mathematical model for the coinfection of buruli ulcer and cholera," Results in Physics, vol. 29, article 104746, 2021.

[17] X.-Y. Wang, K. Hattaf, H.-F. Huo, and H. Xiang, "Stability analysis of a delayed social epidemics model with general contact rate and its optimal control," Journal of Industrial and Management Optimization, vol. 12, no. 4, pp. 1267-1285, 2016.

[18] S. Athithan, M. Ghosh, and X.-Z. Li, "Mathematical modeling and optimal control of corruption dynamics," Asian-European Journal of Mathematics, vol. 11, no. 6, article 1850090, 2018.

[19] X.-P. Li, Y. Wang, M. A. Khan, M. Y. Alshahrani, and T. Muhammad, "A dynamical study of SARS-CoV-2: a study of third wave," Results in Physics, vol. 29, article 104705, 2021.

[20] A. Gumel, Introduction to dynamical system in mathematical epidemiology, Icmcsnuc workshop, Abuja, Nigeria., 2007.

[21] C. Castillo-Chavez, S. Blower, P. Van den Driessche, D. Kirschner, and A.-A. Yakubu, Mathematical Approaches for Emerging and Reemerging Infectious Diseases: An Introduction, Springer Science \& Business Media, 2002.

[22] H. W. Hethcote, "The mathematics of infectious diseases," SIAM Review, vol. 42, no. 4, pp. 599-653, 2000.

[23] F. Benyah, Introduction to epidemiological modeling, 10th regional college on modeling, simulation and optimization, University of cape coast, Ghana, 2008.

[24] J. P. Lasalle, The Stability of Dynamical Systems, SIAM, 1976.

[25] W. H. Fleming and R. W. Rishel, Deterministic and Stochastic Optimal Control, Springer Science \& Business Media, 2012.

[26] W. E. Boyce and R. C. DiPrima, Elementary Differential Equations and Boundary Value Problems, John Wiley \& Sons, Inc., 2012.

[27] E. O. Alzahrani, W. Ahmad, M. Altaf Khan, and S. J. Malebary, "Optimal control strategies of zika virus model with mutant," Communications in Nonlinear Science and Numerical Simulation, vol. 93, article 105532, 2021.

[28] D. L. Lukes, Differential Equations: Classical to Controlled, Mathematics in Science and Engineering, 1982.

[29] V. Boltyanskiy, R. V. Gamkrelidze, Y. Mishchenko, and L. Pontryagin, Mathematical Theory of Optimal Processes, CRC Press, 1962. 